\title{
A NOTE ON THE UNIFORMIZATION OF GRADIENT KÄHLER RICCI SOLITONS
}

\author{
Albert Chau and Luen-Fai Tam
}

\begin{abstract}
Applying a well known result for attracting fixed points of biholomorphisms $[5,7]$, we observe that one immediately obtains the following result: if $\left(M^{n}, g\right)$ is a complete non-compact gradient Kähler-Ricci soliton which is either steady with positive Ricci curvature so that the scalar curvature attains its maximum at some point, or expanding with non-negative Ricci curvature, then $M$ is biholomorphic to $\mathbb{C}^{n}$.
\end{abstract}

In this note we will prove the following:

Theorem 1. If $\left(M^{n}, g\right)$ is a complete non-compact gradient Kähler-Ricci soliton which is either steady with positive Ricci curvature so that the scalar curvature attains its maximum at some point, or expanding with non-negative Ricci curvature, then $M$ is biholomorphic to $\mathbb{C}^{n}$.

In [2], Cao constructed examples of complete rotationally symmetric gradient Kähler-Ricci solitons with positive holomorphic bisectional curvature on $\mathbb{C}^{n}$. Theorem 1 implies that in fact all complete gradient Kähler-Ricci solitons with positive holomorphic bisectional curvature, satsifying the assumptions in the theorem, are defined on $\mathbb{C}^{n}$. The theorem is related to the uniformization conjecture of Yau which states that all complete non-compact Kähler manifolds with positive holomorphic bisectional curvature are biholomorphic to $\mathbb{C}^{n}$ (see [3] for more details).

Recall that a Kähler-Ricci soliton is a solution to the un-normalized KählerRicci flow

$$
\frac{\partial}{\partial t} g_{i \bar{j}}=-R_{i \bar{j}}
$$

which evolves only by dilation and pull back along a one parameter family of biholomorphisms. More specifically, $\left(M, g_{i j}(x)\right)$ is said to be a Kähler-Ricci soliton if there is a family of biholomorphisms $\phi_{t}$ on $M$, given by a holomorphic vector field $V$, such that $g_{i j}(x, t)=\phi_{t}^{*}\left(g_{i j}(x)\right)$ is a solution of the Kähler-Ricci

Received April 2004, Revised July 2004.

2000 Mathematics Subject Classification. Primary 53C44; Secondary 58J37, 35B35.

First author research partially supported by The Institute of Mathematical Sciences, The Chinese University of Hong Kong, Shatin, Hong Kong, China.

Second author research partially supported by Earmarked Grant of Hong Kong \#CUHK4032/02P. 
flow:

$$
\begin{gathered}
\frac{\partial}{\partial t} g_{i \bar{j}}=-R_{i \bar{j}}-2 \rho g_{i \bar{j}} \\
g_{i \bar{j}}(x, 0)=g_{i \bar{j}}(x)
\end{gathered}
$$

for $0 \leq t<\infty$, where $R_{i \bar{j}}$ denotes the Ricci tensor at time $t$ and $\rho$ is a constant. If $\rho=0$, then the Kähler-Ricci soliton is said to be of steady type and if $\rho>0$ then the Kähler-Ricci soliton is said to be of expanding type. We always assume that $g$ is complete and $M$ is non-compact. If in addition, the holomorphic vector field is given by the gradient of a real valued function $f$, then it is called a gradient Kähler-Ricci soliton. Note that in this case, we have that

$$
\begin{aligned}
& f_{i \bar{j}}=R_{i \bar{j}}+2 \rho g_{i \bar{j}} \\
& f_{i j}=0 .
\end{aligned}
$$

If $(M, g)$ is a gradient Kähler-Ricci soliton which is either steady with positive Ricci curvature so that the scalar curvature attains its maximum at some point, or expanding with non-negative Ricci curvature, then one can show that $\phi_{t}$, the flow on $M$ along the vector field $-\nabla f$, satisfies:

(i) $\phi_{t}$ is a biholomorphism from $M$ to $M$ for all $t \geq 0$,

(ii) $\phi_{t}$ has a unique fixed point $p$, i.e. $\phi_{t}(p)=p$ for all $t \geq 0$,

(iii) $\mathrm{M}$ is attracted to $p$ under $\phi_{t}$ in the sense that for any open neighborhood $U$ of $p$ and for any compact subset $W$ of $M$, there exists $T>0$ such that $\phi_{t}(W) \subset U$ for all $t \geq T$.

Condition (i) is clear. Condition (ii) is shown in $[3,4]$. To see that condition (iii) holds, we consider any $R>0$ and let $B(R)$ be the geodesic ball of radius $R$ with center at $p$ with respect to the metric $g(0)$. From the proof of Lemma 3.2 in [3], there exists $C_{R}>0$ such that for any $q \in B(R)$ and for any $v \in T^{1,0}(M)$ at $q$,

$$
\|v\|_{\phi_{t}^{*}(g)} \leq \exp \left(-C_{R} t\right)\|v\|_{g}
$$

Since $\phi_{t}(p)=p$, it is easy to see that given any open set $U \subset M$ containing $p$, we have $\phi_{t}(B(R)) \subset U$ provided $t$ is large, and thus condition (iii) is satisfied.

We now observe a general result on the biholomorphic structure of a basin of attraction for any biholomprphism on a complex manifold. The following theorem was proved for the case $M=\mathbb{C}^{n}$ in [5], and was later observed to be true on a general complex manifold $M$ in [7].

Theorem 2. Let $F$ be a biholomorphism from a complex manifold $M^{n}$ to itself and let $p \in M^{n}$ be a fixed point for F. Fix a complete Riemannian metric $g$ on $M$ and define

$$
\Omega:=\left\{x \in M: \lim _{k \rightarrow \infty} \operatorname{dist}_{g}\left(F^{k}(x), p\right)=0\right\}
$$

where $F^{k}=F \circ F^{k-1}, F^{1}=F$. Then $\Omega$ is biholomorphic to $\mathbb{C}^{n}$ provided $\Omega$ contains an open neighborhood around $p$. 
Proof of Theorem 1. By conditions (i)-(iii) we may apply Theorem 2 to the biholomorphism $\phi_{1}: M \rightarrow M$ to conclude that $M$ is biholomorphic to $\mathbb{C}^{n}$.

Remark 1. In the first version of this article we proved Theorem 2 in a special case. We would like to thank Dror Varolin for pointing out to us that what we proved had been known earlier [5, 7].

Remark 2. After posting the first version of this article we learned that Theorem 1 in the case of a steady gradient Kähler Ricci soliton had been known independently to Robert Bryant [1].

\section{References}

[1] R. Bryant, Gradient Kahler Ricci Solitons, arXiv eprint 2004. arXi:math.DG/0407453

[2] H. D. Cao, Existence of gradient Kähler-Ricci soliton (Minneapolis, MN, 1994), A K Peters, Wellesley, MA, (1996), 1-16. MR98a:53058.

[3] A. Chau, L.-F. Tam, Gradient Kähler-Ricci soliton and a uniformization conjecture, arXiv eprint 2003. arXi:math.DG/0310198.

[4] R. S. Hamilton, Formation of Singularities in the Ricci Flow, Contemporary Mathematics, 71 (1988), 237-261.

[5] J. P. Rosay and W. Rudin, Holomorphic Maps from $\mathbb{C}^{n}$ to $\mathbb{C}^{n}$, Trans. AMS 310 (1988), 47-86.

[6] S. Sternberg, Local contractions and a theorem of Poincar, Amer. J. Math., 79 (1957), 809-824.

[7] D. Varolin, The density property for complex manifolds and geometric structures II, Internat. J. Math. 11 (2000), 837-847.

Harvard University, Department of Mathematics, One Oxford Street, Cambridge, MA 02138, USA

E-mail address: chau@math.harvard.edu

Department of Mathematics, The Chinese University of Hong Kong, Shatin, Hong Kong, China.

E-mail address: lftam@math.cuhk.edu.hk 